\title{
Web-based interactive educational software introducing semiconductor laser dynamics: Sound of Lasers (SOL)
}

Antonio Consoli, Jorge Sanchez, Paloma Horche, Ignacio Esquivias

Antonio Consoli, Jorge Rodero Sanchez, Paloma R. Horche, Ignacio Esquivias, "Web-based interactive educational software introducing semiconductor laser dynamics: Sound of Lasers (SOL)," Proc. SPIE 9289, 12th Education and Training in Optics and Photonics Conference, 92891S (17 July 2014); doi: 10.1117/12.2070560

Event: 12th Education and Training in Optics and Photonics Conference, 2013, Porto, Portugal 


\title{
Web based interactive educational software introducing semiconductor laser dynamics: Sound Of Lasers (SOL)
}

\author{
Antonio Consoli ${ }^{1}$, Jorge Rodero Sanchez ${ }^{2}$, Paloma R. Horche ${ }^{2}$ and Ignacio Esquivias ${ }^{1}$ \\ ${ }^{1}$ CEMDATIC - ETSI de Telecomunicación - Universidad Politécnica de Madrid \\ ${ }^{2}$ Dpto. de Tecnología Fotónica y Bioingeniería - ETSI de Telecomunicación \\ Universidad Politécnica de Madrid \\ Avenida Complutense 32, E-28040, Madrid, Spain \\ antonio.consoli@upm.es
}

\begin{abstract}
In this work, educational software for intuitive understanding of the basic dynamic processes of semiconductor lasers is presented. The proposed tool is addressed to the students of optical communication courses, encouraging self consolidation of the subjects learned in lectures.

The semiconductor laser model is based on the well known rate equations for the carrier density, photon density and optical phase. The direct modulation of the laser is considered with input parameters which can be selected by the user. Different options for the waveform, amplitude and frequency of the injected current are available, together with the bias point. Simulation results are plotted for carrier density and output power versus time.

Instantaneous frequency variations of the laser output are numerically shifted to the audible frequency range and sent to the computer loudspeakers. This results in an intuitive description of the "chirp" phenomenon due to amplitude-phase coupling, typical of directly modulated semiconductor lasers. In this way, the student can actually listen to the time resolved spectral content of the laser output. By changing the laser parameters and/or the modulation parameters, consequent variation of the laser output can be appreciated in intuitive manner.

The proposed educational tool has been previously implemented by the same authors with locally executable software. In the present manuscript, we extend our previous work to a web based platform, offering improved distribution and allowing its use to the wide audience of the web.
\end{abstract}

Keywords: e-learning, educational software, java applets, optical communications, semiconductor lasers, laser rate equations model, frequency chirp.

\section{INTRODUCTION}

Semiconductor lasers (SLs) are fundamental components of fiber based optical communication channels, due to their compact sizes, high reliability and low power operating conditions. Directly modulated (DM) SLs are the preferred choice in short range optical networks, e. g. Local Area Network (LAN) and Metropolitan Area Networks (MANs), due to the reduced system cost and complexity with respect to externally modulated laser sources [1]. Additionally, short and intense optical pulses with duration of tens of picoseconds can be generated with SLs when the driving conditions are properly set in order to work in gain switching [2] regime. Gain Switched (GS) SLs are practical pulse sources that find applications in a wide variety of fields, e. g. telemetry, optical ranging, 3D scanning, material processing and biomedical analysis.

A clear understanding of the basic dynamics processes in SLs is then relevant for the student audience of university photonics courses. In this work we present a web hosted educational tool for the simulation of DM SLs: the device model is based on a simple formulation of the well know Rate Equations (REs) of carrier and photon density and optical phase. The laser internal parameter and current driving conditions can be set by the user and the carrier density and laser output power are plotted as a function of time. Instantaneous frequency variations, typical of DM SLs, are numerically shifted to the audible frequency range and the resulting signal is sent to the computer loudspeakers, giving an intuitive description of the "chirp" phenomenon in SLs [3].

This work is organized as follows. In Section 2, the proposed simulation tool is described, giving details of the mathematical model in use, its numerical implementation and the user control interface. In Section 3, some examples of use are presented, in which the program is run in different conditions, changing the laser modulation parameters. Finally, in Section 4, the main conclusions of the proposed work are summarized.

12th Education and Training in Optics and Photonics Conference, edited by

Manuel F. P. C. Martins Costa, Mourad Zghal, Proc. of SPIE Vol. 9289, 92891S

(c) 2014 SPIE, OSA, IEEE, ICO · doi: 10.1117/12.2070560

Proc. of SPIE Vol. 9289 92891S-1 


\section{SOFTWARE DESCRIPTION}

\subsection{The Rate Equations Model}

The light-matter interactions occurring in a SL can be modeled in a simple way by using the RE model [1, 4].The RE model considers the interplay between carriers and photons in the laser cavity in terms of conservation equations. The number of electrons and holes is assumed to be the same, thus only one equation for carriers is considered in the model. The main assumption of the model is that carriers and photons are considered to be uniformly distributed in the longitudinal and transversal directions.

Assuming a single mode laser, the relationship between the carrier density, $\mathrm{N}(\mathrm{t})$, the photon density, $\mathrm{S}(\mathrm{t})$, and the optical phase, $\phi(\mathrm{t})$, can be expressed by the following equations:

$$
\begin{aligned}
& \frac{d N(t)}{d t}=\frac{I(t)}{q V_{a c t}}-\frac{N(t)}{\tau_{N}}-v_{G} \frac{d G}{d N} \frac{\left(N(t)-N_{0}\right)}{1+\varepsilon S(t)} S(t) \\
& \frac{d S(t)}{d t}=v_{G} \Gamma \frac{d G}{d N} \frac{\left(N(t)-N_{0}\right)}{1+\varepsilon S(t)} S(t)-\frac{S(t)}{\tau_{P}}+\beta \Gamma \frac{N(t)}{\tau_{N}} \\
& \frac{d \phi(t)}{d t}=\frac{\alpha}{2}\left(v_{G} \Gamma \frac{d G}{d N}\left(N(t)-N_{0}\right)-\frac{1}{\tau_{P}}\right)
\end{aligned}
$$

where the descriptions of the symbols and their values are given in Tab. 1. Typical values for a $1550 \mathrm{~nm}$ Distributed Feed Back (DFB) laser [5] are used by default.

The RE model previously described has been implemented in Java ${ }^{\circledR}[6]$ programming language and the resulting applet is hosted by Universidad Politécnica de Madrid, in a web server at Tecnología Fotónica y Bioingeniería (www.tfo.upm.es/educational-tools). Equations 1-3 are solved with a Runge Kutta iterative algorithm for $\mathrm{N}(\mathrm{t}), \mathrm{S}(\mathrm{t})$ and $\phi(\mathrm{t})$, with temporal resolution of $1 \mathrm{ps}$ for a vector of 10000 points $(10 \mathrm{~ns})$.

Table 1. Symbols and default values used in Eqs. 1-3.

\begin{tabular}{|l|l|c|c|}
\hline \multicolumn{4}{|c|}{ 1550 nm DFB Laser [5] } \\
\hline \multicolumn{1}{|c|}{ Symbol } & \multicolumn{1}{|c|}{ Description } & $1.602 \cdot 10^{-19}$ & Units \\
\hline $\mathrm{q}$ & Elementary charge & $2 \cdot 10^{-11}$ & $\mathrm{~cm}^{3}$ \\
\hline $\mathrm{V}_{\text {act }}$ & Active volume & 0.25 & - \\
\hline$\Gamma$ & Confinement factor & $1.7 \cdot 10^{-16}$ & $\mathrm{~cm}^{2}$ \\
\hline $\mathrm{dG} / \mathrm{dN}$ & Differential gain & $9.4 \cdot 10^{9}$ & $\mathrm{~cm}^{-} \mathrm{s}$ \\
\hline $\mathrm{V}_{\mathrm{G}}$ & Group velocity & $1.6 \cdot 10^{18}$ & $\mathrm{~cm}^{-3}$ \\
\hline $\mathrm{N}_{0}$ & Transparency carrier density & 1 & $\mathrm{~ns}$ \\
\hline$\tau_{\mathrm{N}}$ & Carrier lifetime & 1 & $\mathrm{ps}$ \\
\hline$\tau_{\mathrm{P}}$ & Photon lifetime & $1.7 \cdot 10^{-17}$ & $\mathrm{~cm}$ \\
\hline$\varepsilon$ & $\begin{array}{l}\text { Nonlinear gain compression } \\
\text { factor }\end{array}$ & $1 \cdot 10^{-4}$ & - \\
\hline$\beta$ & Spontaneous emission coefficient & 3.5 & - \\
\hline$\alpha$ & Linewidth enhancement factor & & \\
\hline
\end{tabular}

\subsection{The Graphic User Interface}

The Graphic User Interface (GUI) is shown in Fig. 1. It is composed of four panels and three graphs. From the Modulation Parameters panel the user can choose the current waveform, the bias point, the peak-to-peak amplitude and the frequency of the modulating current. The waveform of the current can be selected by the user, either sinusoidal or rectangular.

The laser parameters can be introduced by the user in the Laser Parameters panel, placed on the right side of the GUI. Values shown in Tab. 1 are considered by default. Carrier density threshold $\left(\mathrm{N}_{\mathrm{TH}}\right)$ and threshold current $\left(\mathrm{I}_{\mathrm{TH}}\right)$ are 
calculated as $\mathrm{N}_{\mathrm{TH}}=\mathrm{N}_{0}+\left(\tau_{\mathrm{P}} \cdot \mathrm{dG} / \mathrm{dN} \cdot \mathrm{v}_{\mathrm{G}} \cdot \Gamma\right)^{-1}$ and $\mathrm{I}_{\mathrm{TH}}=\mathrm{N}_{\mathrm{TH}} \cdot \mathrm{q} \cdot \mathrm{V}_{\text {act }} \tau_{\mathrm{N}}{ }^{-1}$, by solving Eqs. 1-2 in steady state regime at threshold.

By pressing the Run button in the Modulation Parameter panel, the program solves Eqs. 1-3, and returns the values of carrier density, photon density and optical phase at each temporal instant. The instantaneous frequency, $v(t)$, is calculated from the temporal derivative of the phase, as $v(t)=(2 \pi)^{-1} \mathrm{~d} / \mathrm{dt}(\phi(t))$. Three graphs on the left side of the GUI show the results of the simulation. From up to down, the temporal profiles of injected current, carrier density and output power are shown. Current and carrier density are plotted as a function of $\mathrm{I}_{\mathrm{TH}}$ and $\mathrm{N}_{\mathrm{TH}}$, respectively.

From the laser intensity and chirp, the software builds an amplitude and frequency modulated signal of the form $A(t) \cdot \cos \left(2 \pi\left(f_{0}+f(t)\right)\right.$, where $A(t)$ is proportional to $S(t), f(t)$ is the frequency $v(t)$ shifted into the audible frequency range. This conversion is obtained with a constant factor of $10^{9}$, changing nanoseconds into seconds and $\mathrm{GHz}$ into $\mathrm{Hz}$. The Sound control is placed at the bottom of the right side of the GUI and allows switching on and off the reproduced sound. The Save Data panel allows the user to export the simulation results to a text file, where the time, current, number of carriers, output power and instantaneous frequency vectors are stored in a tab separated format.
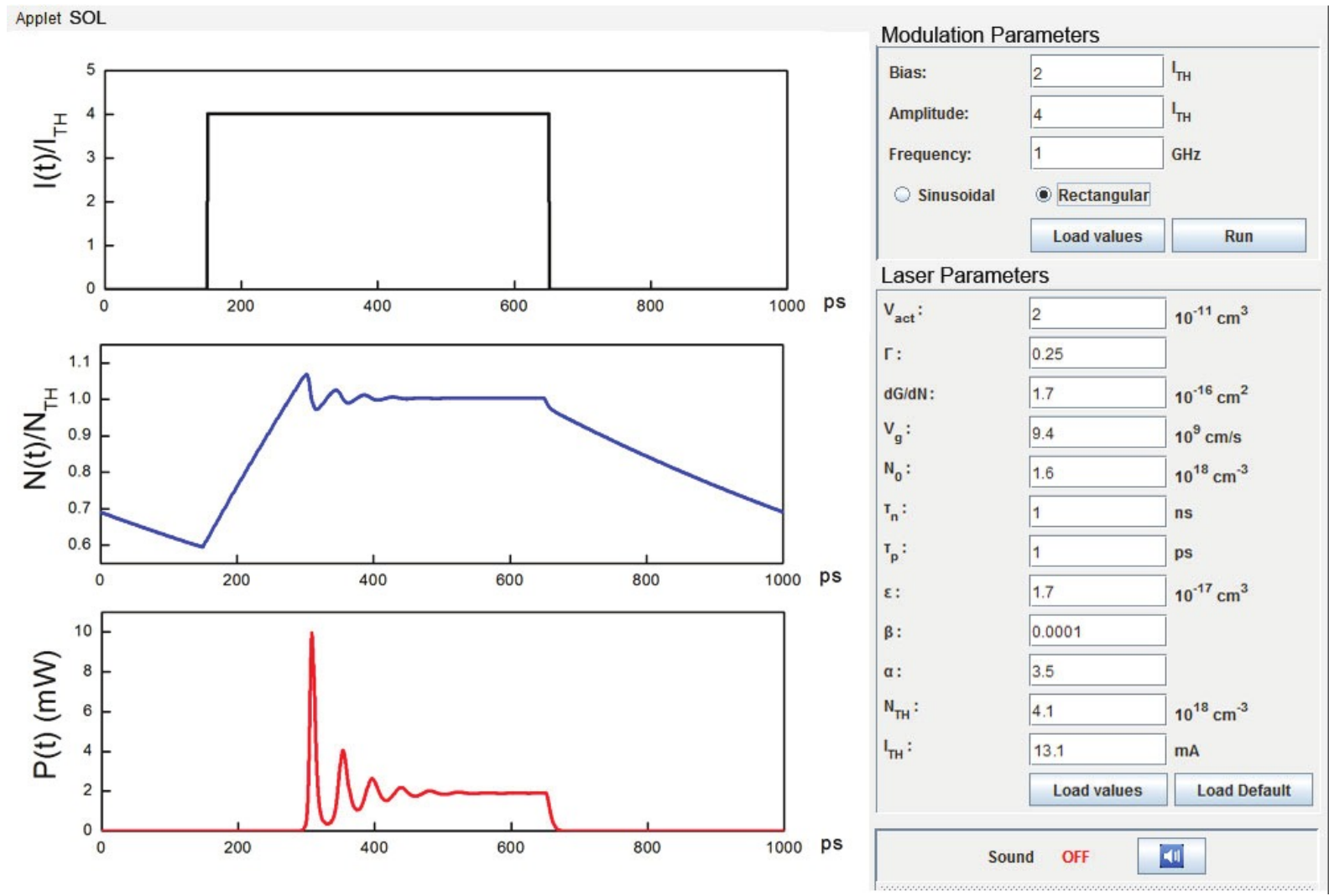

Figure 1. The Graphic Unit Interface.

\section{EXAMPLES}

In this Section, we present some simulation results that can be useful for understanding the dynamics of a DM SL. We show the dependence of the laser output, in terms of intensity and instantaneous frequency, on its internal parameters and on the modulation conditions, i. e. current waveform, current amplitude and bias point. In the proposed examples, attention is given both to the graphical representation of the results and on the impact of the audible output on the user understanding of the simulated phenomenon.

These examples are directed to a student audience with previous basic notions on the theory of laser dynamics. Here we explain how to use the proposed simulation tool and its potential for a better learning experience, giving a brief theoretical description of the simulated phenomena. Results are exported with the Save Data button and presented in figures. 


\subsection{Laser Switch On}

We first consider the simple case in which the SL is switched on with a rectangular current function. In order to have this, in the Modulation Parameters panel, the rectangular current waveform is selected, $\mathrm{I}_{\mathrm{BIAS}}$ is set to $1.5 \mathrm{I}_{\mathrm{TH}}$, the amplitude is set to $3 \mathrm{I}_{\mathrm{TH}}$ and the frequency is set to $0.25 \mathrm{GHz}$ (4 ns period with $50 \%$ duty cycle).

(a)

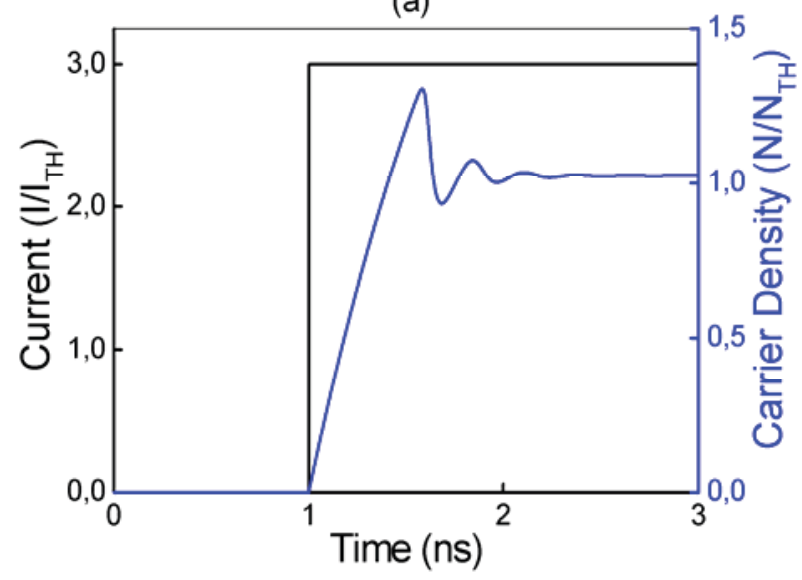

(b)

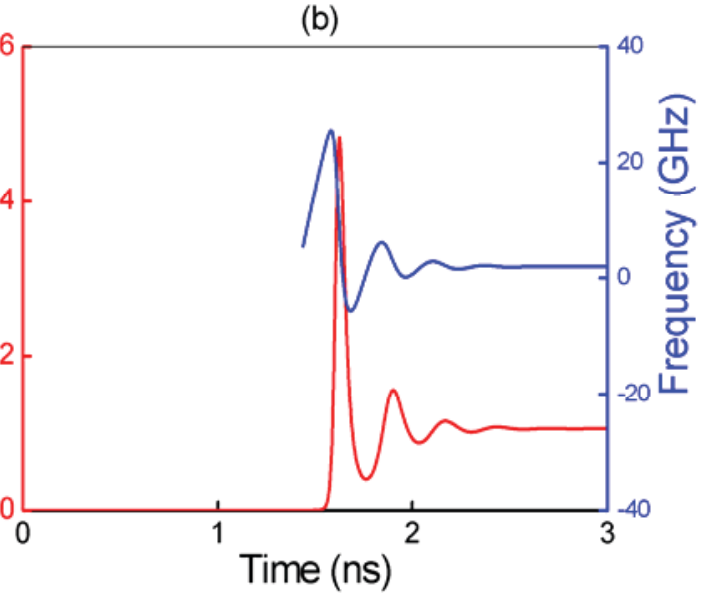

Figure 2. Results obtained with the rectangular current waveform selected, bias set to 0 and amplitude 3 ITH: (a) current and carrier density, black line left axis and blue line right axis, respectively and (b) output power and chirp, red line left axis and blue line right axis, respectively. The time axis is zoomed $3 \mathrm{~ns}$ around the laser switch on.

This corresponds to inject the laser with a current step function with low level equal to 0 and a high level current of $3 \mathrm{I}_{\mathrm{TH}}$. The laser parameters are set to the default values.

After the Run button is pressed, the program takes some seconds to complete the simulation and the results are finally shown in the GUI graphs. Current, carrier density, output power and instantaneous frequency are exported with the Save Data button and results are plotted in Fig. 2 (a) and (b).

When the rectangular current is applied, the carrier density grows as 1- $\exp \left(-\mathrm{t} / \tau_{\mathrm{N}}\right)$ from the low level steady state to $\mathrm{N}_{\mathrm{TH}}$, as expected from theory $[1,4]$. $\mathrm{N}(\mathrm{t})$ undergoes damped oscillations around the threshold density value before reaching the steady state value clamped at $\mathrm{N}_{\mathrm{TH}}$. This corresponds to the relaxation oscillations of the output power, due to carrier induced gain modulation, and of the instantaneous frequency, due to the carrier induced index modulation.

The user can appreciate in an intuitive manner, the coupled amplitude/frequency modulation, by pressing the Sound in the GUI. As expected, when the laser is off, for $\mathrm{I}(\mathrm{t})=0$, no sound is produced, then a chirped sound is emitted, when power and instantaneous frequency oscillate, and finally a single frequency tone is produced, corresponding to the optical carrier of the laser shifted into the audible band.

\subsection{Direct Modulation}

In this example, we consider the direct modulation of the laser with a periodic rectangular current waveform, with the low and high current level both above the threshold current value $\mathrm{I}_{\mathrm{TH}}$. In the Modulation Parameters panel, the rect current waveform is selected, BIAS is set to $4 \mathrm{I}_{\mathrm{TH}}$, the amplitude is set to $2 \mathrm{I}_{\mathrm{TH}}$ and the frequency field is set to $0.5 \mathrm{GHz}$.

After pressing the Run button, the results are plotted in the GUI graphs. In this case, the well known phenomenon of transient and adiabatic chirp in DM SLs is clearly appreciated after listening to the audible output. The transient chirp is usually referred to the instantaneous frequency variation associated to the transition between the two levels of the injected current, i. e. the damped oscillations of $v(t)$ which follows from low to high and from high to low current level transitions. The adiabatic chirp is the frequency difference between the frequency produced during the high level and the frequency produced during the low level of injected current. The adiabatic chirp is directly related to the gain compression factor $\varepsilon$. As shown in Fig. 3 (a) and (b), the carrier density is not clamped to the threshold value NTH as in the ideal case $(\varepsilon=0)$.

In the proposed simulation, the audio output is composite of a single higher tone during the high level state, a lower single tone during the low level state (corresponding to the adiabatic chirp) and a chirped sound when the current switches from low to high level and viceversa (corresponding to the transient chirp). This is also appreciated from the 
instantaneous frequency temporal profile, where the frequency difference between low and high level of the injected current due to the adiabatic chirp, is clearly shown in Fig. 4 (a) and (b). If the gain compression factor $\varepsilon$ is set to zero, the adiabatic chirp signature disappears from the audio output and the graphs, as expected from theory.

(a)

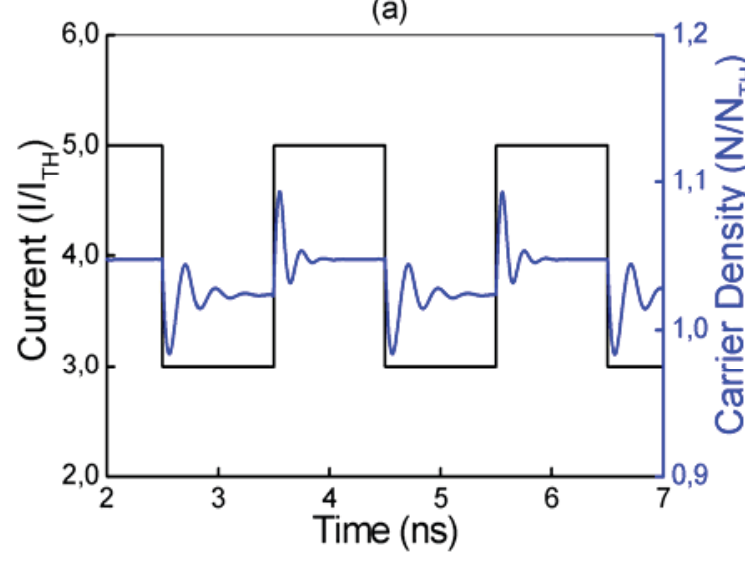

(b)

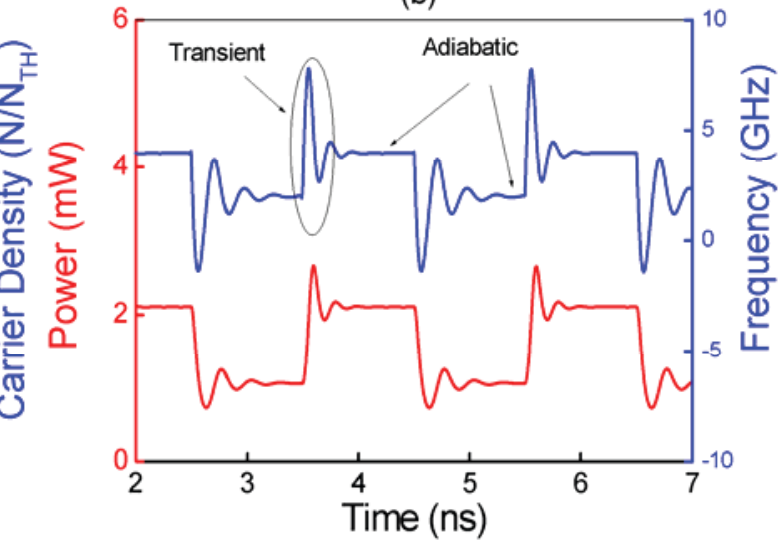

Figure 3. Results obtained with the rectangular current waveform selected, bias set to $4 \mathrm{I}_{\mathrm{TH}}$, amplitude $2 \mathrm{I}_{\mathrm{TH}}$ and frequency 0.5 GHz: (a) current and carrier density, black line left axis and blue line right axis, respectively and (b) output power and chirp, red line left axis and blue line right axis, respectively.

\section{CONCLUSIONS}

We have presented novel educational software for the simulation of the dynamics of directly modulated semiconductor lasers. The proposed tool is directed to undergraduate students of photonics and optical communications courses.

The software is based on the well known RE model for SLs, and it is numerically implemented on a Java ${ }^{\circledR}$ applet. The GUI allows the student to choose the laser internal parameters and the modulating conditions. The simulation results are plotted and the amplitude/frequency modulation typical of current injected SLs is shifted to the audible frequency range for an intuitive understanding of the phenomenon. Several examples are given, i. e. the laser turn on and laser direct modulation, and results are discussed for different modulation parameters.

The proposed software allows the user to explore and test his knowledge on SL dynamics with an interactive tool by changing the operating condition of the simulated device and see and listen to the consequent results in the laser output.

\section{REFERENCES}

[1] G. P. Agrawal, [Fiber-Optic Communication Systems], John Wiley \& Sons, Inc., 2002

[2] K. Y. Lau, "Gain switching of semiconductor injection lasers" Appl. Phys. Lett. 52 (4), 257-259 (1988)

[3] A. Consoli, B. Bonilla, P. R. Horche and I. Esquivias "Sound of lasers (SOL) - An audiovisual approach to semiconductor laser dynamics", Proc. of the 15th International Conference on Interactive Collaborative Learning (ICL), 26 - 28 September 2012, Villach, Austria.

[4] L. A. Coldren and S. W. Corzine, [Diode Lasers and Photonic Integrated Circuits], John Wiley \& Sons, Inc., 1995.

[5] John C. Cartledge and R. C. Srinivasan, "Extraction of DFB Laser Rate Equation Parameters for System Simulation Purposes”, J. Lightwave Technol., vol. 15, iss. 5, pp. 852-860, 1997.

[6] www.oracle.com

\section{ACKNOWLEDGEMENTS}

This work has been supported by Universidad Politécnica de Madrid, through the project "Desarrollo de técnicas de aprendizaje y metodología activas para la impartición de la Fotónica". We gratefully acknowledge the support of the Ministerio de Economia y Competitividad (Spain) through project TEC2012-38864-C03-02. 